\title{
Water Vapor Detection System Based on Scanning Spectra
}

\author{
Shicong ZHANG ${ }^{1}$, Qiang WANG ${ }^{1}$, Yan $Z \mathrm{ZHANG}^{1}$, Fujun $\mathrm{SONG}^{1}$, Kun $\mathrm{CHEN}^{2}$, \\ Guoqing $\mathrm{CHOU}^{1}$, Jun $\mathrm{CHANG}^{1}$, Pengpeng $\mathrm{WANG}^{1}$, Delong KONG ${ }^{1}$, Zongliang WANG ${ }^{1}$, \\ Weijie WANG ${ }^{1}$, Yongning $\mathrm{LIU}^{1}$, and Haiyong $\mathrm{SONG}^{1}$
}

\author{
${ }^{1}$ School of Information Science and Engineering and Shandong Provincial Key Laboratory of Laser Technology and \\ Application, Shandong University, Jinan, 250100, China \\ ${ }^{2}$ School of Shandong Provincial Party Committee of Communist Party of China, Jinan, 250021, China \\ *Corresponding author: Jun CHANGＥ-mail: changjun@sdu.edu.cn
}

\begin{abstract}
Scanning the absorption spectral line of water vapor through wavelength around $1368.597 \mathrm{~nm}$ is successfully used to measure the value of micro-moisture content. The synchronous superposition average of original signal algorithm based on labview is innovated and applied to detecting weak spectrum absorption signal instead of low pass filter. Two data processing methods are used to get the concentration of water vapor in ppm: one is a general formula method which has newly deduced a general formula to calculate the concentration of gas with temperature and beam intensity ratio when the pressure is equal to or greater than $1 \mathrm{~atm}$; the other is engineering calibration method which is proved to have high resolution and accuracy with the fitted curve of beam intensity ratio and concentration in ppm when the temperature changes form $258 \mathrm{~K}$ to $305 \mathrm{~K}$ and the pressure ranges from $1 \mathrm{~atm}$ to $5 \mathrm{~atm}$.
\end{abstract}

Keywords: Water-vapor detection, scanning spectra, detecting under high gas pressure, near-infrared absorption

\section{Introduction}

As is known to all, water, the earth's most widely distributed and reserved substance that exists in three forms as gas, liquid and solid, has been closely related to human activities. Moisture measurement, previously mainly used for meteorological observation, has now been inseparable from many fields such as industry [1], agriculture, and information technology [2]. Looking only at industry, it contains metallurgy, petroleum, chemical [3], electronics, electric power, textile, medicine, foodstuff, timber, papermaking, architecture, air conditioning, and even the nuclear and aerospace industries. In this century, water measurement technology has been developed rapidly by creating various types of hygrometer and humidity sensor for measuring water in the three forms. Moisture analysis can be classified into two major categories. One is physical analysis method including weightlessness method [4], distillation method [5], adsorption method [6], relative density method, dew point method [7], electro-analysis, spectrometry, gas chromatography [8], etc. The other is chemical analysis method consisting of gas escape tube evolution method, acid or alkali generation method, Karl Fischer method [9], etc. However, these methods have some shortages and limitations in practical application: on the one hand, tasting results always vary from water vapor measuring

Received: 20 May 2011 / Revised version: 24 June 2011

(C) The Author(s) 2011.This article is published with open access at Springerlink.com 
devices to testing technology which is sensitively influenced not merely by real-time temperature and humidity but also by artificial error from operation; on the other hand, detecting period is very long to lead a potential risk of equipment and inefficient site tracing monitoring. Therefore, with its high efficiency, practicality, property of environmental protection, spectrometry has been widely applied and developed in the field of gas detection.

Difference method [10] and second harmonic method [11] are the current research focus on detecting water vapor concentration using spectrometry. Nevertheless, not only has difference method its limited measuring accuracy, but also second harmonic method needs complicated signal processing. In this paper, scanning the absorption spectral line of water vapor around $1369 \mathrm{~nm}$ to measure its concentration is used for the first time. A near infrared (NIR) distributed feedback laser diode (DFB-LD) $[12,13]$ is used as light source of which wavelength changes along with its injected current. The water vapor concentration can be calculated using the beginning and the transmitted laser intensities [14]. This scheme has quicker response, higher sensitivity and better accuracy than previous ones.

\section{Measurement principle}

Once transitions happen, spectral lines will appear owing to a gas molecule containing large amounts of rotation and vibration levels. Normally, transitions of these two kinds of levels occur at the same time, thus generating NIR spectra which are widely used in engineering applications [15].

The water vapor spectrum in the region of $1368.51 \mathrm{~nm}-1368.69 \mathrm{~nm}$ with its center of $1368.597 \mathrm{~nm}$ is analyzed to be the best absorption transition for sensitive measurement of concentration according to HITRAN database [16] when the temperature $(T)$ is $296 \mathrm{~K}$ and the pressure $(P)$ is $1 \mathrm{~atm}$ for the reasons as follows:

(1) The region of $1368.51 \mathrm{~nm}-1368.69 \mathrm{~nm}$ includes only one transition so that there will be no chance for that transition to overlap with neighboring transitions when the pressure broadening [17] complicates absorption measurement and introduces time-varying absorption transition line widths.

(2) The transition, near $1368.6 \mathrm{~nm}$, is selected as a particularly promising line due to the superior signal-to-noise ratio (SNR) in the later circuit.

(3) Compared with other wave band, tunable diode laser around $1330 \mathrm{~nm}$ has many strongpoints such as higher reliability, mature technology and less fiber loss in single mode of about $0.27 \mathrm{~dB} / \mathrm{km}$ [18].

In direct absorption spectroscopy measurements, a collimated laser beam with an intensity of $I_{0}$ is shone through the sample gas, and the transmitted laser intensity $I$ is measured with a detector, as shown in Fig. 1. When the laser frequency $v\left(\mathrm{~cm}^{-1}\right)$ is resonant with the frequency of a transition for the absorbing species in the gas, the laser energy will be absorbed. The attenuation of the laser intensity along the path length of $L$ can be described as follows:

$$
\left(I / I_{0}\right)=\exp (-\alpha(v) \cdot P \cdot L)
$$

where $P(\mathrm{~atm})$ is the total pressure, $L(\mathrm{~cm})$ is the gas path length, $\alpha(v)\left(\mathrm{atm}^{-1} \cdot \mathrm{cm}^{-1}\right)$ is the absorption coefficient. $\left(I / I_{0}\right)$ (intensity ratio) can be measured along with the scanning spectra.

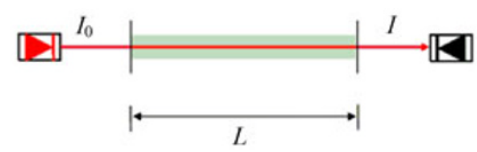

Fig. 1 Schematic of Beer-Lambert.

From the formulae mentioned in [19-23], we deduce the following general formula to calculate the concentration of gas with temperature and intensity ratio when $P$ is equal to or greater than $1 \mathrm{~atm}$ for the first time:

$C=\frac{\gamma_{\text {others }}(T)}{\gamma_{\text {others }}(T)-\gamma_{\text {self }}(T)+\left(1-\left(I / I_{0}\right)_{v, \min }\right)^{-1} \cdot \frac{L \cdot S(T)}{K \cdot \pi \cdot T \times 10.1325}} \times 10^{6}$

where $K, 1.3806505 \times 10^{-23}(\mathrm{~J} / \mathrm{K})$, is Boltzmann 
constant, $T$ (Kelvin) is the gas temperature, $\left(I / I_{0}\right)_{v \cdot \min }$ is the minimum of intensity ratio, $C$ is the concentration of the detected gas in ppm (percent per million), $S(T)\left(\mathrm{cm} \cdot \mathrm{mol}^{-1}\right)$ is the line strength of the transition at temperature $T, \gamma_{\text {self }}(T)\left[\mathrm{cm}^{-1} \cdot \mathrm{atm}^{-1}\right]$ and $\gamma_{\text {others }}(T) \quad\left(\mathrm{cm}^{-1} \cdot \mathrm{atm}^{-1}\right)$ are the broadening coefficients due to the collisions between the absorbing molecules themselves and between the absorbing molecules and the perturbing molecules. $S(T), \gamma_{\text {self }}(T)$ and $\gamma_{\text {others }}(T)$ can be found in HITRAN database. However, in addition, we can just calculate the water vapor concentration in air at present with (2) as only the broadening coefficient due to the collisions between the absorbing molecules and the air molecules $\left(\gamma_{\text {others }}(T)=\gamma_{\text {air }}(T)\right)$ can be checked out according to the database.

\section{Experimental facility}

Figure 2 shows a schematic of the experimental arrangement for the quantitative measurement of water vapor concentration. The length of gas cell is $10 \mathrm{~cm}$. A DFB-LD is used as primary laser source to investigate $\mathrm{H}_{2} \mathrm{O}$ spectra near $1369 \mathrm{~nm}$. The fiber-coupled output of the light source is split into two beams $(0.9,0.1)$. The $90 \%$ intensity is transmitted through the sample gas, caught and sent to an INGaAs PIN (positive-intrinsic-negative) photo detector. The rest $10 \%$ intensity is transmitted through fiber to a similar detector to provide an intensity reference. Both signals from two detectors are collected by data acquisition card (DAC) which is connected to a computer to do further processing. The output power of the $90 \%$ intensity varies with wavelength and must be normalized using the reference signal for quantitative absorption measurement in order to remove the laser intensity fluctuations.

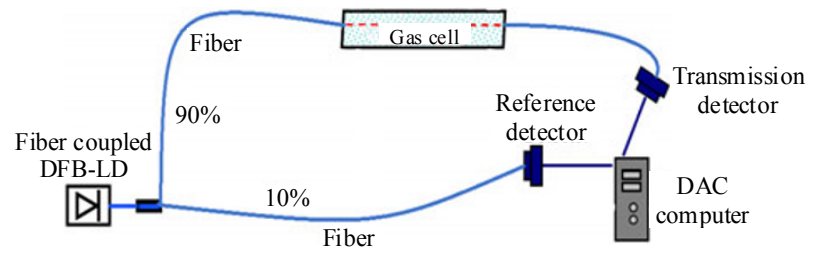

Fig. 2 Experimental configuration.

\section{Concluding water vapor concentration by general formula}

This test is conducted under 1 atm at the temperature of $287 \mathrm{~K}$ when the water vapor concentration of air in ppm is 20.67 .

As shown in Fig. 3, the "I" area which is not absorbed by the water vapor and the "II" area around $1368.59 \mathrm{~nm}$ whose absolute value is attenuated because of gas absorption are both included in one scanning period. In Figs. 4 and 5, curve $\mathrm{C}$ is transmitted intensity signal; curve $\mathrm{D}$ is reference signal; curve $\mathrm{A}$, the normalized curve (beam intensity ratio, BIR for short), get filtered in matlab, thus obtaining curve $\mathrm{B}$; curve $\mathrm{E}$ is normalized signal processed by synchronous superposition average algorithm in labview.

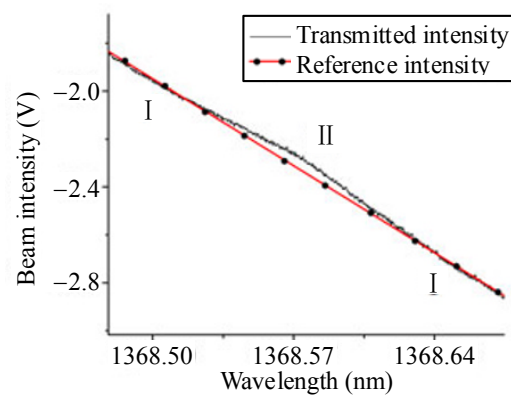

Fig. 3 Spectral intensity along with wavelength.

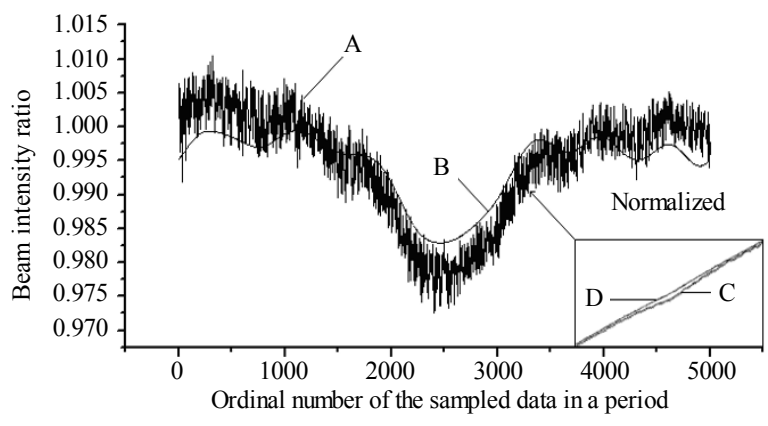

Fig. 4 Filtering the normalized signal.

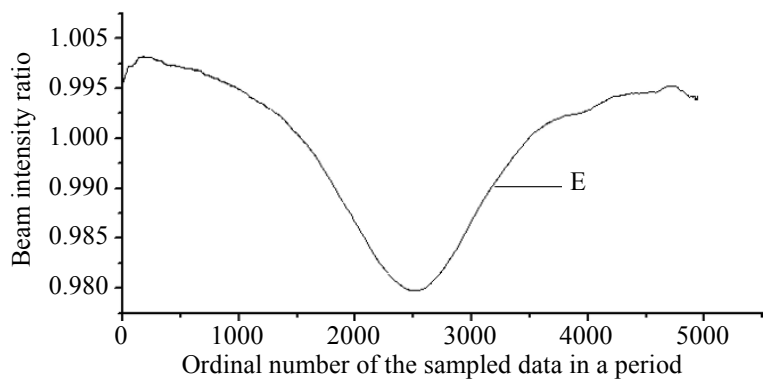

Fig. 5 Normalized signal processed by synchronous superposition average algorithm. 
Obviously, curve A needs further treatment for its large noise. However, curve B, processed by matlab low pass filter, is always found to have lower peak value than curve $A$, even the low-pass frequency and the type of filters (including Butterworth, Chebyshev, Elliptic, and Equiripple) have been replaced several times. Therefore, low pass filter is not a good choice for this test. In order to solve this problem, we designed a synchronous superposition average algorithm which theoretically took the average value of multiple stacking signals collected several times under the same circumstance. This method can offset all kinds of random noise and filter out disturbed noises effectively (for example, the positive and negative noises at a certain wavelength point share the same proportion of $50 \%$ for random character when the stacking fold tends to be infinite, once being superimposed and averaged, they will be completely cancelled out. At the same time, the useful signals accumulate constantly, which can lead to a good signal to noise ratio. However, restricted by collection rate and hardware performance, the work efficiency reduces as the superposition number increases. Therefore, we need to determine the superposition number according to the demand of system precision. We set the stacking number around 200 times after repeated experiments and the corresponding result was shown as curve $\mathrm{E}$ which has the same line shape that exactly matched curve A. Taking the peak value of curve E of 0.97898 into (2) and calculating the concentration in ppm, we found the outcome in line with the real value detected by dew point hygrometer.

According to the theory of this algorithm, the error is only concerned with superposition number. In order to get relatively more accurate standard value, we took 24 hours to set the number as 1209600 , thus getting the peak value of curve $\mathrm{E}$ of 0.97895 . Therefore, by comparing 0.97898 with $0.97895,200$ times superposition number was acceptable in this experiment.

\section{Concluding water vapor concentration by calibration method}

The transmitted intensity (I) will have little change in contrast to $I_{0}$ if the mixed gas has extremely low water vapor concentration, thus making little change in DAC distortion range and leading an inaccurate peak value of the BIR. In order to handle this problem, we should improve SNR of the BIR before the signal is caught by DAC (shown in Fig. 6). Consequently, the final peak value ( $W_{\min }$ for short) of signal processing is not the original BIR anymore because of basic operation induced by a series of analog devices. Instead of figuring out the peak value of BIR from $W_{\min }$ and calculating the concentration with (2), we designed a new method of calibration as it was difficult to obtain the inverse calculation coefficient.

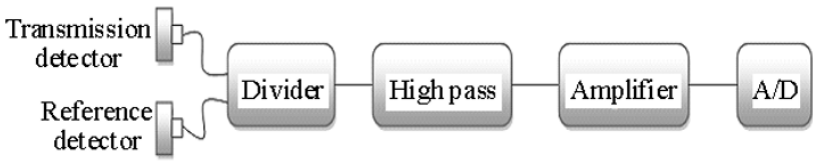

Fig. 6 Signal processing.

This test was conducted when the temperature changed form $258 \mathrm{~K}$ to $305 \mathrm{~K}$ and the pressure ranged from $1 \mathrm{~atm}$ to $5 \mathrm{~atm}$. The final peak values, in turn, were recorded in Table 1 when changing the water vapor concentration, temperature and pressure in the gas cell. The objective concentrations in ppm were written down according to specula-surface condensation dew point instrument at the same time, thus providing data to fit a function curve $f\left(W_{\min }\right)$ (shown in Fig. 7) reflecting the rule that objective concentration in ppm varied from $W_{\min }$. During this experiment, we found that if the concentration was determined, $W_{\min }$ would remain invariant even the pressure and temperature kept changing when the pressure was greater than or equal to $1 \mathrm{~atm}$ and the temperature ranged from $258 \mathrm{~K}$ to $305 \mathrm{~K}$. However, just to be complete, calibration should be updated 
since the pressure would not affect $W_{\min }$ unless the pressure is below $1 \mathrm{~atm}$.

Table 1 Recorded and calculated data.

\begin{tabular}{ccccc}
\hline Group & $W_{\min }$ & $f\left(W_{\min }\right)$ & $\begin{array}{c}\text { Objective } \\
\text { concentration }\end{array}$ & Difference $(\%)$ \\
\hline 1 & -2.41008 & 14.892 & 19.01634 & -21.6884 \\
2 & -2.40335 & 45.00875 & 50.4610 & -10.8049 \\
3 & -2.40086 & 56.1515 & 61.5549 & -8.77818 \\
4 & -2.38591 & 123.0528 & 125.985 & -2.32746 \\
5 & -2.38234 & 139.0285 & 140.1574 & -0.80545 \\
6 & -2.36072 & 235.778 & 220.299 & 7.02636 \\
7 & -2.35569 & 258.2872 & 251.954 & 2.513653 \\
8 & -2.34773 & 293.9083 & 300.313 & -2.13269 \\
9 & -2.31726 & 430.2615 & 430.684 & -0.0981 \\
10 & -2.27513 & 618.7933 & 600.246 & 3.089941 \\
11 & -2.18046 & 1042.442 & 1042.33 & 0.010697 \\
12 & -2.11209 & 1348.397 & 1345.56 & 0.21086 \\
13 & -2.00013 & 1849.418 & 1865.27 & -0.84984 \\
\hline & & & &
\end{tabular}

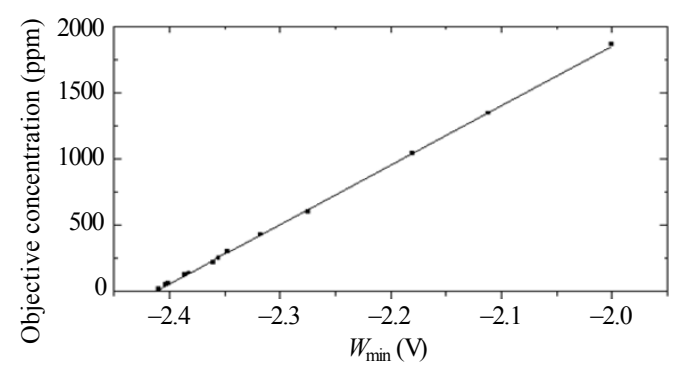

Fig. 7 Acquired data points and fitted curve.

In Fig. 7, $W_{\min }$ is abscissa, objective concentration in ppm is vertical coordinate, the filled dots come from Table 1, and the fitted curve is linear expressed as (3):

$$
f\left(W_{\min }\right)=4475 \cdot W_{\min }+1.083 \times 10^{4} .
$$

Once the function of $f\left(W_{\min }\right)$ is confirmed, the real concentration in ppm can be calculated by taking $W_{\min }$ into (3).

Table 1 makes a thorough exposition on the difference between the objective concentration and the concentration in ppm operated with (3). The relative error increases when the concentration gets decreased for the follow reasons:

(1) The concentration is too low for specula-surface condensation dew point instrument to perform as well as expected.

(2) It is difficult to process low SNR signal caused by quite low water vapor concentration.

In calibration method, $11 \mathrm{ppm}$ concentration can be picked out (groups 2 and 3 in Table 1) and the measurement error basically stays within a stable level of $10 \mathrm{ppm}$.

\section{Conclusions}

The water vapor detection system based on scanning spectra has properties of high efficiency, practicality, sensitivity and low cost. It can be realized by two kinds of means: general formula method and calibration method. Each method has its own advantages and disadvantages: general formula method, easy manipulated, does not suit for the circumstances blow the pressure of $1 \mathrm{~atm}$ and mixed gases apart from air; calibration method, complex for large amounts of scale data, has higher sensitivity. Finally, by means of synchronous superposition average of original signal algorithm, both of these two schemes successfully explored and validated the possibility of high precision measurement of water vapor concentration through scanning spectra around $1369 \mathrm{~nm}$.

\section{Acknowledgment}

This work was supported by Natural Science Foundation of China (60977058), Science Fund for Distinguished Young Scholars of Shandong Province of China (JQ200819), Research Award Fund for Outstanding Middle-aged and Young Scientist of Shandong Province of China (2007BS08003), Independent Innovation Foundation of Shandong University (IIFSDU2010JC002).

Open Access This article is distributed under the terms of the Creative Commons Attribution License which permits any use, distribution, and reproduction in any 
medium, provided the original author(s) and source are credited.

\section{References}

[1] G. P. Li, J. N. Chen, D. F. Huang, and G. Jie, "Real-time monitoring system of precipitable water vapor derived from ground-based GPS and its applications in meteorology," Geomatics and Information Science of Wuhan University, vol. 34, no. 11, pp. 1328-1331, 2009 (in Chinese).

[2] X. Ren and D. Yang, "Analysis and comparison of moisture content in electronic components," Microelectronics, vol. 39, no. 5, pp. 718-721, 2009 (in Chinese).

[3] X. Xue, J. B. Duan, W. Q. Liu, et al., "Differential optical absorption spectroscopy measurement for flue gas $\mathrm{SO}_{2}$," Journal of Atmospheric and Environmental Optics, vol.3, no. 2, pp. 121-124, 2008 (in Chinese).

[4] Z. Q. Chen and W. F. Wei, "Study on the determination of water in solid dispersion of ursolic acid and PEG6000," Strait Pharmaceutical Journal, vol. 21, no. 10, pp.15-17, 2009 (in Chinese).

[5] W. J. Lin, R. Q. Xu, and Y. M. Zhang, "GC-MS analysis of chemical constituents of the essential oil from plumula nelumbinis by different extraction methods," Chinese Journal of Pharmaceutical Analysis, vol. 29, no. 11, pp. 1858-1862, 2009 (in Chinese).

[6] J. Liu, "Study on purification of small amount of water from organic solvent by adsorption," M.S. thesis, Nanjing University of Technology, Nanjing, China, 2006 (in Chinese).

[7] J. Zhang, G. Y. Chou, B. S. Guo, and T. P. Guo, "Dewpoint-based judgement of endpoint of primary sublimation in freeze-drying process," Vacuum, no. 4, pp. 66-71, 2009 (in Chinese).

[8] L. P. Zhu, T. Zhu, Y. X. Pan, J. Sun, and J. Dong, "Determination of different kinds of pesticide residues in vegetable by gas-chromatography," Chinese Journal of Analytical Chemistry, vol. 36, no. 7, pp. 999-1003, 2008 (in Chinese).

[9] R. Meng and R. T. Shang, "The development and application of Karl Fischer method for determining water," Chemical Reagents, vol. 23, no. 1, pp. 39-41, 2001 (in Chinese).

[10] Y. Q. Sun and X. T. Lu, "Research on improving sensitivity of the characteristic spectrum in micro-gas monitor," Spectroscopy and Spectral Analysis, vol. 30, no. 7, pp. 1890-1893, 2010 (in Chinese).

[11] S. Schilt, "Impact of water vapor on $1.51 \mu \mathrm{m}$ ammonia absorption features used in trace gas sensing applications," Appl. Phys. B, vol. 100, no. 2, pp. 349-359, 2010.

[12] R. U. Martinelli, R. 1. Menna, D. E. Cooper, C. B. Carlisle, and H. Riris, "Near-infrared InGaAs/Inp distributed feedbacklasers for spectroscopic applications," SPIE, vol. 2148, pp. 292307, 1994.

[13] V. Weldon, J. O'Gorman, and P. Phelan, "Gas sensing with $\lambda=1.57 \mu \mathrm{m}$ distributed feedback laser diodes using overtone and combination band absorption," Optic. Eng., vol. 33, no. 12, pp. 3867-70, 1994

[14] W. Jin, G. Cooper, C. B. Carlise, and H. Riris, "Absorption measurement of methane gas with a broadband light sourceand interferometric signal processing," Optics Letters, vol. 18, no. 16, pp. 1364-1366, 1993.

[15] Y. C. Wei, B. Y. Wu, L. Y. Yang, and D. Xing, "Upconversion fluorescence monitoring near-infrared during tumor photothermal therapy," Chinese Journal of Lasers, vol. 37, no. 11, pp. 2719-2724, 2010 (in Chinese).

[16] L. S. Rothman, D. Jacquemart, A. Barbe, et al., "The HITRAN 2004 molecular spectroscopic database," J. Quant. Spectrosc. Radiat. Transfer, vol. 96, no. 2, pp. 139-204, 2005.

[17] A. Ray, A. Bandyopadhyay, B. Ray, D. Biswas, and P. N. Ghosh, "Line-shape study of water vapor by tunable diode laser spectrometer in the $822-832 \mathrm{~nm}$ wavelength region," Appl. Phys. B, vol. 79, no. 7, pp. 915-921, 2004

[18] R. J. Zhang, U. X. Zheng, J. Li, L. Y. Chen, and C. F. Ge, "The dispersion and attenuation measurement system for single mode optical fiber," Research and Exploration In Laboratory, vol. 28, no. 10, pp. 29-31, 2009 (in Chinese).

[19] X. Liu, J. B. Jeffries, and R. K. Hanson, "Measurements of spectral parameters of water-vapour transitions near 1388 and $1345 \mathrm{~nm}$ for accurate simulation of high-pressure absorption spectra," Meas. Sci. Technol., vol. 18, no. 5, pp. 1185-1194, 2007.

[20] H. Tsukioka and K. Sugawara, "New apparatus for detecting transformer faults," IEEE Transactions on Electrical Insulation, vol. 21, no. 2, pp. 24-35, 1986.

[21] R. R. Gamache, S. Kennedy, R. Hawkins, and L. S. Rothman, "Total internal partition sums for molecules in the terrestrial atmosphere," Mol. Struct., vol. 517-518, pp.407-425, 2000.

[22] J. S. Gibb, G. Hancock, A. Hutchinson, R. Peverall, and G. A. D. Ritchie, "On the accurate determination of pressure induced line shifts in the $2 \vee 3$ band of H2O at 1320 nm," Appl. Phys. B, vol. 76, no. 1, pp. 97-105, 2003.

[23] W. G. Ma, W. B. Yi, T. Huang, et al., "Analysis of gas absorption coefficient at various pressures," Spectroscopy and Spectral Analysis, vol. 24, no. 2, pp. 135-137, 2004. 